Florida International University FIU Digital Commons

$11-15-2002$

\title{
Association between mean residual life (MRL) and failure rate functions for continuous and discrete lifetime distributions
}

Leonid Bekker

Florida International University

DOI: $10.25148 /$ etd.FI14050479

Follow this and additional works at: https://digitalcommons.fiu.edu/etd

Part of the Statistics and Probability Commons

\section{Recommended Citation}

Bekker, Leonid, "Association between mean residual life (MRL) and failure rate functions for continuous and discrete lifetime distributions" (2002). FIU Electronic Theses and Dissertations. 1479.

https://digitalcommons.fiu.edu/etd/1479 


\section{FLORIDA INTERNATIONAL UNIVERSITY \\ Miami, Florida}

ASSOCIATION BETWEEN MEAN RESIDUAL LIFE (MRL) AND FAILURE RATE FUNCTIONS FOR CONTINUOUS AND DISCRETE LIFETIME DISTRIBUTIONS

A thesis submitted in partial fulfillment of the

requirements for the degree of

MASTER OF SCIENCE

in

STATISTICS

by

Leonid Bekker 
To: Dean Arthur W. Herriott

College of Arts and Sciences

This thesis, written by Leonid Bekker, and entitled Association Between Mean Residual Life (MRL) and Failure Rate Functions for Continuous and Discrete Lifetime Distributions, having been approved in respect to style and intellectual content, is referred to you for judgment.

We have read this thesis and recommend that it be approved.

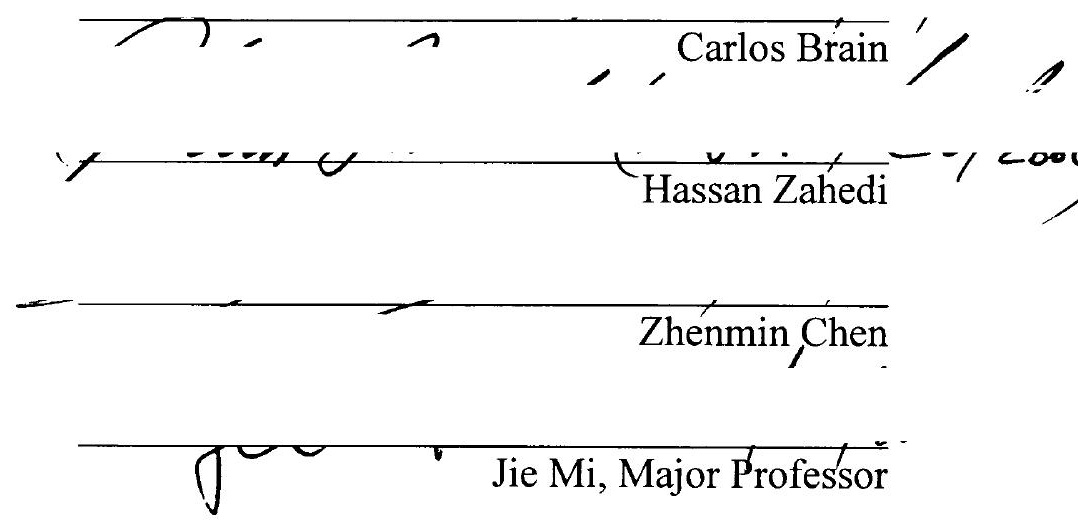

Date of Defense: November 15, 2002

The thesis of Leonid Bekker is approved.

Dean Arthur W. Herriott College of Arts and Scheptes

Dean Dfuglas Wartzok
University Graduate School

Florida International University, 2002 


\section{DEDICATION}

I dedicate this thesis to my father, who is no longer with us and sadly cannot share this special moment with me and my mother, who always provides me with a great deal of support, and who believes that I can achieve anything I am willing to work hard for. 


\section{ACKNOWLEDGMENTS}

I wish to thank the members of my committee for their support, patience, and guidance, especially I want to thank my major professor, Dr. Jie Mi. Without his great support and encouragement this work would never have come into existence. He continuously set an example of how dedication and commitment can lead to success. 


\begin{abstract}
OF THE THESIS
ASSOCIATION BETWEEN MEAN RESIDUAL LIFE ( MRL) AND FAILURE RATE FUNCTIONS FOR CONTINUOUS AND DISCRETE LIFETIME DISTRIBUTIONS
\end{abstract}

by

Leonid Bekker

Florida International University, 2002

Miami, Florida

Professor Jie Mi, Major Professor

The purpose of this study was to correct some mistakes in the literature and derive a necessary and sufficient condition for the MRL to follow the roller-coaster pattern of the corresponding failure rate function. It was also desired to find the conditions under which the discrete failure rate function has an upside-down bathtub shape if corresponding MRL function has a bathtub shape. The study showed that if discrete MRL has a bathtub shape, then under some conditions the corresponding failure rate function has an upside-down bathtub shape. Also the study corrected some mistakes in proofs of Tang, Lu and Chew (1999) and established a necessary and sufficient condition for the MRL to follow the roller-coaster pattern of the corresponding failure rate function. Similarly, some mistakes in Gupta and Gupta (2000) are corrected, with the ensuing results being expanded and proved thoroughly to establish the relationship between the crossing points of the failure rate and associated MRL functions. The new results derived in this study will be useful to model various lifetime data that occur in environmental studies, medical research, electronics engineering, and in many other areas of science and technology. 


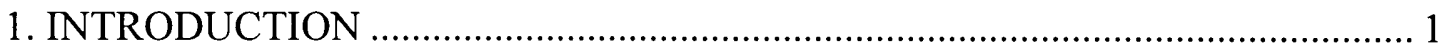

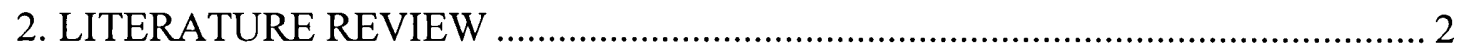

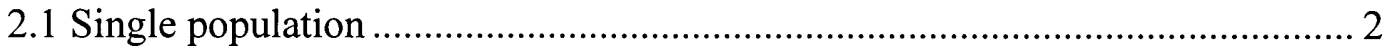

2.1.1 Bathtub and upside-down bathtub failure rate functions ..................... 2

2.1.2 Bathtub and upside-down bathtub MRL functions ............................... 5

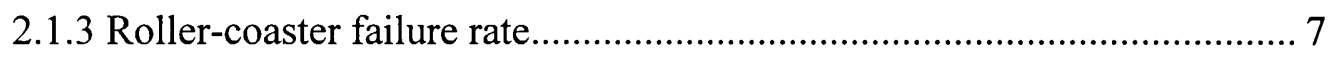

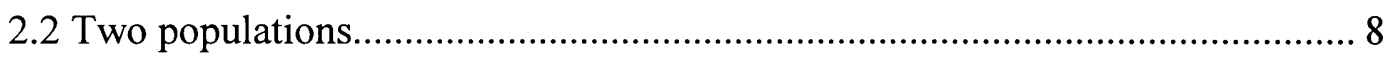

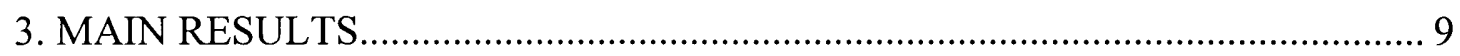

3.1 Discrete bathtub MRL and upside-down bathtub failure rate functions ........... 9

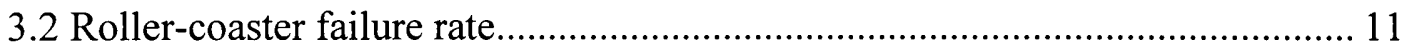

3.3 Crossing of the failure rate and MRL functions ............................................ 19

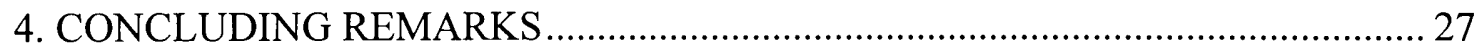

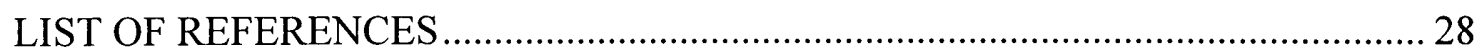




\section{Introduction}

Reliability theory and its applications deal with distributions of continuous and discrete lifetimes. The two commonly used measures of describing the lifetime of items are the mean residual life (MRL) function and the failure rate function.

Lifetime distributions having decreasing, increasing, bathtub-shaped, or upside-down bathtub-shaped MRL are used to model various lifetime data which occur in many areas of science such as reliability, survival analysis, economics, actuarial, and many others. For example, in biomedical sciences, researchers analyze survivorship studies by using the MRL (see, e.g., Gupta (1981)). The failure rate function also has numerous applications, including modelling the lifetime of electronic, electro-mechanical, and mechanical products. For example, Mi (1996) discussed useful models for improving the quality of products after they have been produced, if the failure rate function of the products exhibits a bathtub shape. On the other hand, upside-down bathtubshaped failure rate functions, typified by failure due to fatigue, are commonly used for modelling lifetimes of mechanical parts and semiconductors (see Peck and Zerdt (1974)).

Since both the MRL and failure rate functions are extremely important for characterizing lifetime distributions and are theoretically equivalent, in the sense that knowledge of one of them determines that of the other, the relationship between these measures have been extensively studied in the literature. In this paper, we further knowledge on the relationship of these two functions. The organization of this paper is as follows. Section 2 provides an extensive review of the most important 
results regarding the general behavior of the MRL, for both continuous and discrete lifetime distributions with respect to their failure rate functions, and vise versa. The main results of this paper are presented in Section 3. A new result on the relationship between the bathtub-shaped MRL function and the upside-down bathtub-shaped failure rate function is derived in Section 3.1. In Section 3.2, some mistakes in the proofs of Tang, Lu and Chew (1999) are corrected, and a necessary and sufficient condition for the MRL to exhibit the roller-coaster shape is established. In Section 3.3, some mistakes in Gupta and Gupta (2000) are corrected, and the results are expanded and proved thoroughly to show that the number of crossings of the MRL functions do not exceed the number of crossings of the failure rate functions. Finally, some concluding remarks appear in Section 4.

\section{Literature Review}

\subsection{Single Population}

\subsubsection{Bathtub and Upside-Down Bathtub Failure Rate Function}

For continuous and discrete cases, it has been shown that the shape of the MRL function can be inferred from its failure rate.

\section{Continuous Case}

The relationship between the bathtub-shaped failure rate function and the upsidedown bathtub-shaped MRL function was established in Mi (1995). He proved that the MRL of a component has an upside-down bathtub shape if the component has a bathtub-shaped failure rate function, but the converse does not hold. This result 
shows that a proper burn-in process maximizes the MRL of a product if its failure rate function has a bathtub shape.

Theorem 2.1. Let $F$ have a differentiable bathtub-shaped failure rate function $r(t)$ with change points $t_{1}$ and $t_{2}$. Then the following statements are true:

(i). If $0<t_{1} \leq t_{2}<\infty$, then the mean residual life function $\mu(t)$ has an upside-down bathtub shape with a unique change point $t_{0} \in\left[0, t_{1}\right]$;

(ii). If $0=t_{1} \leq t_{2}<\infty$, then $\mu(t)$ strictly decreases, i.e., there is a unique change point at $t=0$;

(iii). If $t_{1}=t_{2}=\infty$, then $\mu(t)$ strictly increases;

(iv). If $0<t_{1}<t_{2}=\infty$, then $\mu(t)$ strictly increases on $\left[0, t_{1}\right]$ and is constant on $\left[t_{1}, \infty\right]$.

Proof. See Mi (1995).

This result implies that if the failure rate has a bathtub shape, then the associated MRL has an upside-down bathtub shape.

Tang, Lu and Chew (1999) discussed the relationship between upside-down bathtubshaped failure rate and bathtub-shaped MRL functions. Such characterization provides useful information for planning spare provision, formulating warranty policy, and some other applications (see Siddiqui and Caglar (1994)). It was shown there, that under some necessary condition, the MRL has a bathtub shape if the failure rate function has an upside-down bathtub shape. The result was provided by the following theorem. 
Theorem 2.2. If $F$ has a differentiable upside-down bathtub-shaped failure rate function $r(t)$ with a change point $t_{0}$, then for $\mu(0) \cdot r(0) \leq 1$, the corresponding MRL has a bathtub shape with change point $t_{1} \in\left[0, t_{0}\right]$; otherwise, it is an increasing $M R L$ for all $t \geq 0$.

Proof. See Tang, Lu and Chew (1999).

\section{Discrete Case}

The relationship between bathtub-shaped failure rate and upside-down bathtub-shaped MRL functions has been investigated by Mi (1993). It follows, under some conditions, that the MRL has an upside-down bathtub shape if the failure rate function has a bathtub shape. This result could be applied for optimization of the burn-in process in order to obtain the longest MRL in field operation.

Theorem 2.3. If a discrete lifetime distribution function $F$ has support set $\{1,2, \ldots\}$, and the failure rate sequence $\{r(i), i \geq 1\}$ has a bathtub shape with change points $n_{1}$ and $n_{2}<\infty$, then for the MRL sequence $\{\mu(i), i \geq 1\}$, there are three cases :

(i). If the probability mass function $p(1)<1 /(1+\mu)$, then $\mu(i)$ strictly decreases;

(ii). If $p(1)=1 /(1+\mu)$, then $\mu(1)=\mu(2)>\mu(3)>\ldots$;

(iii). If $p(1)>1 /(1+\mu)$, then $\{\mu(i), i \geq 1\}$ has an upside-down bathtub shape with either a unique change point $k_{0}$, or two change points $k_{0}-1$ and $k_{0}$, where $k_{0} \leq n_{1}$ in either case.

Proof. See Mi (1993). 
The result, that gives some sufficient conditions under which the MRL has a bathtub shape if the failure rate function has an upside-down bathtub shape, was provided by Tang, Lu and Chew (1999).

Theorem 2.4. Let function $F$ be a lifetime CDF having support set $\left\{t_{1}, t_{2}, \ldots\right\}$, where $t_{1}=0$. Let the failure rate sequence $\left\{r\left(t_{i}\right), i \geq 1\right\}$ have an upside-down bathtub shape with a change point $t_{l}, l \geq 1$. Then, for the $M R L$ sequence $\left\{\mu\left(t_{i}\right), i \geq 1\right\}$, there are three cases :

(i). If $p(0)>1 /(1+\mu)$, then $\mu\left(t_{i}\right)$ strictly increases;

(ii). If $p(0)=1 /(1+\mu)$, then $\mu\left(t_{1}\right)=\mu\left(t_{2}\right)<\mu\left(t_{3}\right)<\ldots$;

(iii). If $p(0)<1 /(1+\mu)$, then $\left\{\mu\left(t_{i}\right), i \geq 1\right\}$ has a bathtub shape with a unique change point $t_{k}, k=m_{0}+1 \leq l$, or two change points $t_{k_{1}}$ and $t_{k_{2}}, k_{1}=m_{0}$ and $k_{2} \leq l$, where $m_{0} \equiv \sup \left\{i \geq 1: s\left(t_{i}\right) \leq 0\right\}$ and $S\left(t_{i}\right) \equiv \frac{p\left(t_{i}\right)}{R\left(t_{i}\right)} \int_{t_{i+1}}^{\infty} R(t) d(t)-R\left(t_{i}\right)$.

Proof. See Tang, Lu and Chew (1999).

\subsubsection{Bathtub and Upside-Down Bathtub MRL Functions.}

The estimation of the MRL function is much more stable than the estimation of the failure rate function, since the estimation of the failure rate involves estimation of the probability density function. Therefore, in many applications, it is desirable to use information provided by the MRL function to check whether the underlying distribution has an upside-down bathtub or bathtub shape property. 


\section{Continuous Case}

Ghai and Mi (1999) developed sufficient conditions for the upside-down bathtubshaped MRL to imply a bathtub-shaped failure rate function. The result is as follows.

Theorem 2.5. Let a unimodal (upside-down bathtub-shaped) $M R L \mu(t)$ have a unique change point $t_{0}$. Suppose there exists $\tau_{0} \in\left[t_{0}, \infty\right)$ such that $\mu(t)$ is concave on $\left[0, \tau_{0}\right)$ and convex on $\left[t_{0}, \infty\right)$. If $\mu^{\prime}(t)$ is convex on $\left[t_{0}, \tau_{o}\right)$, then :

(i). $\quad r(t)$ exhibits a bathtub shape that has two change points, say $t_{1}$ and $t_{2}$, where $t_{0} \leq t_{1}<t_{2} \leq \tau_{0}$

or

(ii). $\quad r(t)$ exhibits a bathtub shape that has a unique change point, say $t^{*}$, where $t_{0} \leq t^{*} \leq \tau_{0}$

Proof. See Ghai and Mi (1999).

Conversely, Ghai and Mi (1999) also developed sufficient conditions for the bathtubshaped MRL with a unique change point $t_{0}$ to imply an upside-down bathtub-shaped failure rate function.

Theorem 2.6. Let $t_{0}$ be the unique change point of a buthtub-shaped MRL function. Suppose there exists $\tau_{0} \in\left[t_{0}, \infty\right)$ such that $\mu(t)$ is convex on $\left[0, \tau_{0}\right)$ and concave on $\left[t_{0}, \infty\right)$. If $\mu^{\prime}(t)$ is concave on $\left[t_{0}, \tau_{o}\right)$, then either the following $(i)$ or $(i i)$ is true for the $r(t)$ function associated with $\mu(t)$ :

(i). $\quad r(t)$ exhibits an upside-down bathtub shape that has two change points, say $t_{1}$ and $t_{2}$, where $t_{0} \leq t_{1}<t_{2} \leq \tau_{0}$. 
(ii). $r(t)$ exhibits an upside-down bathtub shape that has a unique change point, say $t^{*}$, where $t_{0} \leq t^{*} \leq \tau_{0}$.

Proof. See Ghai and Mi (1999).

\section{Discrete Case}

Mi (1993) developed a sufficient condition to show that an upside-down bathtubshaped MRL could imply a bathtub-shaped failure rate function. The condition was given in the following theorem.

Theorem 2.7. Let the MRL sequence $\{\mu(i), i \geq 1\}$ have an upside-down bathtub shape with a unique change point $t_{0}$, and let the sequence $\{\triangle \mu(i), i \geq 1\}$, where $\triangle \mu(i) \equiv \mu(i+1)-\mu(i)$, have a bathtub shape with a change point $t_{0}+1$. Then the sequence $\{r(i), i \geq 1\}$ of failure rates of $F$ has a bathtub shape with a unique change point $t_{0}$ or $t_{0}+1$, or two change points $t_{0}$ and $t_{0}+1$.

Proof. See Mi (1993).

Nothing was mentioned about the relationship between bathtub-shaped MRL and associated upside-down bathtub-shaped failure rate functions. We develop sufficient conditions under which this result holds. The result is given in Section 3.1.

\subsubsection{Roller-Coaster Failure Rate Functions.}

Tang, Lu and Chew (1999) investigated the behavior of the MRL function for when the associated failure rate function has a roller-coaster shape. A general characterization of the MRL and failure rate relationship was given: for the roller-coaster failure rate, the associated MRL function is the composition of the MRL corresponding to 
the three basic failure rates (decreasing, increasing, and constant failure rate) under some conditions. The result was provided by the following theorem.

Theorem 2.8. Let $F$ have a roller-coaster failure rate with consecutive change points $t_{i}, i=1,2, \ldots, n$, where $0=t_{1}<t_{2}<t_{3}<\cdots<t_{n}=\infty$. For change points $t_{i-1}$ and $t_{i}, \quad 2 \leq i \leq n-1$, let $\left[\mu\left(t_{i-1}\right) \cdot r\left(t_{i-1}\right)-1\right]\left[\mu\left(t_{i}\right) \cdot r\left(t_{i}\right)-1\right] \leq 0$.

Then, $F$ have a MRL which is a combination of the MRL functions corresponding to decreasing failure rate and increasing failure rate with consecutive change points $t_{i}^{*}, i=1,2, \ldots, n-1$, where

$$
0 \leq t_{1}^{*}<t_{2}^{*}<t_{3}^{*}<\cdots<t_{n-1}^{*}=\infty \text { and } t_{i}^{*} \in\left[t_{i}, t_{i-1}\right] \text { for } i=1,2, \ldots, n-2 .
$$

However, their result is not quite correct and the proof is also problematic. In Section 3.2, we correct the errors in the proof of Theorem 2 in Tang, Lu and Chew (1999) and give a new proof.

\subsection{Two Population}

There are many practical situations that call for comparing two groups or treatments, where the two failure rates cross at several points. For example, Rocock et al. (1982) have observed this phenomenon in connection with prognostic studies in the treatment of breast cancer. Champlin et al. (1983) and Begg et al. (1984) have also reported instances of the superiority of a treatment being shortlived. This phenomenon of crossing hazards also arises in the study of frailty models or environmental models (see R. Gupta and C.Gupta (1986), Hougard (1984) and Omori and Johnson (1983)). R. Gupta and C.Gupta (2000) presented a result on the crossing of failure rate functions and associated MRL functions. The obtained result is stated next. 
Theorem 2.9. If two failure rate functions $r(t)$ and $r^{*}(t)$ cross at the points $t_{1}^{*}, t_{2}^{*}, \ldots, t_{k}^{*}$ such that $0=t_{0}^{*}<t_{1}^{*}<\ldots<t_{k}^{*}<\infty$, then the corresponding MRL functions $\mu(t)$ and $\mu^{*}(t)$ cross at most at $k$ points and at most one crossing point occurs in each of the intervals $\left(t_{i}^{*}, t_{i+1}^{*}\right), i=0,1,2, \ldots, k-1$.

This result was based on the claim that MRL functions cannot have the same crossing point as corresponding failure rate functions, that is $\mu^{*}\left(t_{i}^{*}\right) \neq \mu\left(t_{i}^{*}\right)$. It can be shown that this claim is incorrect. The counterexample is provided in Section 3.3.

\section{Main Results}

\subsection{Discrete Bathtub MRL and Upside-Down Bathtub Failure Rate Func- tions}

Theorem 3.1. Let $\{\mu(i), i \geq 1\}$ be a mean residual life sequence for a discrete life time distribution $F$. Suppose $\{\mu(i), i \geq 1\}$ have a bathtub shape with a unique change point $t_{0}$ and the sequence $\{\triangle \mu(i), i \geq 1\}$, where $\triangle \mu(i) \equiv \mu(i+1)-\mu(i)$, have an upside-down bathtub shape with change point $t_{0}$. Then, the sequence $\{r(i), i \geq 1\}$ of failure rates of $F$ have an upside-down bathtub shape with a unique change point $t_{0}-1$ or $t_{0}$, or two change points $t_{0}-1$ and $t_{0}$.

Proof. The MRL function $\mu(t)$ is defined as

$$
\mu(t) \equiv E(T-t \mid T \geq t)
$$

and for the discrete case we have

$$
\mu(i)=\frac{\int_{i}^{\infty} \bar{F}(t) d(t)}{\bar{F}(i-1)}
$$


Thus,

$$
\begin{aligned}
\triangle \mu(i)=\frac{p(i) \int_{i+1}^{\infty} \bar{F}(t) d(t)-\bar{F}^{2}(i)}{\bar{F}(i) \bar{F}(i-1)} & =r(i) \cdot \frac{\int_{i+1}^{\infty} \bar{F}(t) d(t)}{\bar{F}(i)}-\frac{\bar{F}(i)}{\bar{F}(i-1)} \\
& =r(i) \cdot \mu(i+1)-[1-r(i)]
\end{aligned}
$$

where $p(i) \equiv P(T=i)$ is a probability mass function.

From (3.1), it follows that

$$
r(i)=\frac{[\mu(i+1)-\mu(i)]+1}{\mu(i+1)+1}=\frac{\triangle \mu(i)+1}{\mu(i+1)+1} .
$$

Hence,

$$
\begin{aligned}
r(i+1)-r(i) & =\frac{\triangle \mu(i+1)+1}{\mu(i+2)+1}-\frac{\triangle \mu(i)+1}{\mu(i+1)+1} \\
& =\frac{\mu(i+2) \triangle^{2} \mu(i)-(\Delta \mu(i+1))^{2}-\Delta \mu(i)}{[\mu(i+2)+1][\mu(i+1)+1]}
\end{aligned}
$$

where

$$
\triangle^{2} \mu(i) \equiv \triangle \mu(i+1)-\triangle \mu(i)
$$

Define

$$
S(i) \equiv \mu(i+2) \triangle^{2} \mu(i)-(\Delta \mu(i+1))^{2}-\Delta \mu(i) .
$$

Then, from (3.3), it is obvious that the sign of the difference $\Delta r(i)=r(i+1)-r(i)$ is the same as the sign of $S(i)$ for all $i \geq 1$. For any $i \geq t_{0}$, we have $\Delta \mu^{2}(i)<0$, since the sequence $\{\triangle \mu(i), i \geq 1\}$ have an upside-down bathtub shape with change point $t_{0}$. At the same time, $i \geq t_{0}$ implies $\triangle \mu(i)>0$, since $\{\mu(i), i \geq 1\}$ have a bathtub shape with change point $t_{0}$. Hence, $S(i)<0$ for $i \geq t_{0}$, and this implies

$$
r(i+1)-r(i)<0, \quad \forall \quad i \geq t_{0}
$$


Therefore,

$$
r\left(t_{0}\right)>r\left(t_{0}+1\right)>\ldots
$$

Consequently, the failure rate $\{r(i)\}$ is strictly decreasing in $i \geq t_{0}$.

If $i \leq t_{0}-2$, then similarly to (3.4), we can obtain

$$
\Delta^{2} \mu(i)>0 \quad \forall \quad i \leq t_{0}-1
$$

Note that by $(3.2), \mu(i)$ has to satisfy

$$
\triangle \mu(i)+1>0 \quad \forall \quad i \geq 1
$$

Because $\quad \Delta \mu(i)+1>0, \quad \Delta \mu(i+1)<0, \quad$ and $\quad \Delta^{2} \mu(i)>0, \quad \forall \quad 1 \leq i \leq t_{0}-2$, it follows that

$$
-(\Delta \mu(i+1))^{2}-\Delta \mu(i)=-\Delta \mu(i+1)[\Delta \mu(i+1)+1]+\Delta^{2} \mu(i)>\Delta^{2} \mu(i)>0
$$

Considering (3.3), (3.5), and (3.7), we obtain

$$
r(i+1)-r(i)>0, \quad \forall \quad i \leq t_{0}-2
$$

Therefore,

$$
r(1)<r(2)<\ldots<r\left(t_{0}-1\right)
$$

Consequently, the failure rate sequence $\{r(i)\}$ is strictly increasing in $1 \leq i \leq t_{0}-1$. Hence, if $r\left(t_{0}\right)>r\left(t_{0}-1\right)$, then the failure rate sequence $\{r(i), i \geq 1\}$ have an upsidedown bathtub shape with a unique change point $t_{0}$; if $r\left(t_{0}\right)<r\left(t_{0}-1\right)$, then there is a unique change point $t_{0}-1$; and if $r\left(t_{0}\right)=r\left(t_{0}-1\right)$, the sequence have two change points $t_{0}-1$ and $t_{0}$. 


\subsection{Roller-Coaster Failure Rate Function}

As mentioned in Section 2, if the failure rate function have a bathtub shape, then the corresponding MRL function may have an upside-down bathtub shape.

The bathtub-shaped failure rate function is a combination of DFR and IFR functions. In practice, due to various reasons such as (i) the changing hazard conditions; (ii) wear out of items with flaws; (iii) the effect of flaw/defect distribution; and (iv) the test and inspection limits, the failure rate function could be alternatively increasing or decreasing. This is the so-called roller-coaster failure rate. For the detailed description of the physical basis for the roller-coaster failure rate, we refer to K.Wong $(1988,1989,1991)$. In order to develop our study more formally, we first give the following definition.

Definition 1. Let $\varphi(t)$ be a function on $[0, \infty)$. Suppose that there exist points $0<t_{1}<t_{2}<\cdots<t_{k}<\infty$ such that on each of the intervals $\left[t_{j-1}, t_{j}\right], \quad 1 \leq j \leq k+1$, where $t_{0} \equiv 0, t_{k+1} \equiv \infty$ and $\left[t_{k}, t_{k+1}\right]=\left[t_{k}, \infty\right)$, the function $\varphi(t)$ is strictly monotone and it have opposite monotonicity in any two adjacent such intervals. Then, we say that $\varphi(t)$ have a roller-coaster shape with change points $\left\{t_{1}, \cdots, t_{k}\right\}$.

Remark 1. Suppose $\varphi(t)$ is a failure rate function. In Definition 1 it is required that $t_{1}>0$. This requirement will eliminate IFR and DFR from the set of failure rate functions with roller-coaster shape. Even though this requirement is unnecessary, it will give us a great convenience in the discussion below since it can avoid repeated tedious explanation of certain exceptional possibilities. 
Remark 2. Definition 1 does not allow any constant part of $\varphi(t)$ in each interval $\left[t_{j-1}, t_{j}\right], 1 \leq j \leq k+1$. Again, this is not necessary, but for the sake of convenience we will require strict monotonicity of $\varphi(t)$ in each interval $\left[t_{j-1}, t_{j}\right]$.

Remark 3. If $\varphi(t)$ have a roller-coaster shape with change points $\left\{t_{1}, \cdots, t_{k}\right\}$ and $\varphi(t)$ is differentiable, then obviously $\left\{t_{1}, \cdots, t_{k}\right\}$ are also the critical points of $\varphi(t)$, i.e., $\varphi^{\prime}\left(t_{j}\right)=0, \quad 1 \leq j \leq k$.

Tang, Lu and Chew (1999) gave a result pertinent to roller-coaster failure rate functions. For easy reference, we restate it with some modification of notation as follows:

“Let $F(t)$ have a roller-coaster shape failure rate $r(t)$ with change points $\left\{t_{1}, \cdots, t_{k}\right\}$. If $\left[\mu\left(t_{j-1}\right) r\left(t_{j-1}\right)-1\right]\left[\mu\left(t_{j}\right) r\left(t_{j}\right)-1\right] \leq 0,1 \leq j \leq k$, then the MRL of $F(t)$ have a roller-coaster shape with change points $\left\{t_{1}^{\prime}, \cdots, t_{k-1}^{\prime}\right\}$, where $0 \leq t_{1}^{\prime}<t_{2}^{\prime}<\cdots<$ $t_{k-1}^{\prime}<t_{k}^{\prime}=\infty$ and $t_{j}^{\prime} \in\left[t_{j-1}, t_{j}\right], \quad 1 \leq j \leq k$

Tang, Lu and Chew used mathematical induction on $k$, the number of change points, to prove their result. However, their proof is problematic. To see this, let us restate part of their proof:

"Let the result be true for $k=n-1 . \cdots$. Now show that it is true for $k=n$. The failure rate has $n$ consecutive change points, $0=t_{0}<t_{1}<\cdots<t_{n}<t_{n+1}=\infty$. According to the assumption for the failure rate with $n-1$ change points, for $t \leq t_{n-1}$, the MRL of $F$ is the composition of the MRL with respect to DFR and IFR, ..." The mistake is that on the interval $\left[t_{n-1}, \infty\right)=\left[t_{n-1}, t_{n}\right) \cup\left[t_{n}, \infty\right)$, the failure rate $r(t)$ is not strictly monotone and this violates the original assumption of roller-coaster shape. As a matter of fact, the monotonicity of $r(t)$ on $\left[t_{n-1}, t_{n}\right)$ and $\left[t_{n}, \infty\right)$ should 
be different according to the definition of roller-coaster-shaped function. Therefore, the induction assumption cannot be applied to $F(t)$ at this time, and so the proof provided there is incorrect.

We will now give a more complete discussion of the shape of the MRL when the underlying failure rate have a roller-coaster shape. The error in the proof in Theorem 2 of Tang, Lu and Chew (1999) will also be corrected.

Lemma 3.2. Suppose the failure rate function $r(t)$ is differentiable and strictly monotone on $(\tau, \infty)$. Then

$1^{0} . \mu(t)$ does not have a critical point on $[\tau, \infty)$;

$2^{0}$. If $r(t)$ strictly increases on $[\tau, \infty)$, then $\mu(t)$ strictly decreases in $t \geq \tau ;$ if $r(t)$ strictly decreases on $[\tau, \infty)$, then $\mu(t)$ strictly increases in $t \geq \tau$.

Proof. The MRL function $\mu(t)$ is given as

$$
\mu(t)=\frac{\int_{t}^{\infty} \bar{F}(s) d s}{\bar{F}(t)}
$$

Following the result of Mi (1995), define

$$
\mu^{\prime}(t)=\frac{A(t)}{\bar{F}(t)}
$$

where

$$
A(t)=r(t) \int_{t}^{\infty} \bar{F}(s) d s-\bar{F}(t)
$$

and

$$
A^{\prime}(t)=r^{\prime}(t) \int_{t}^{\infty} \bar{F}(s) d s
$$


Now, assume $r(t)$ strictly increases in $t>\tau$. For any $t \geq \tau$, from (3.9), it holds that

$$
\begin{aligned}
A(t) & =\int_{t}^{\infty} r(t) \bar{F}(s) d s-\bar{F}(t) \\
& <\int_{t}^{\infty} r(s) \bar{F}(s) d s-\bar{F}(t) \\
& =\int_{t}^{\infty} f(s) d s-\bar{F}(t)=0 .
\end{aligned}
$$

That is, $\mu^{\prime}(t)<0, \quad \forall t \geq \tau$. This shows that $\mu(t)$ is strictly decreasing in $t \geq \tau$. The result when $r(t)$ strictly decreases on $(\tau, \infty)$ can be similarly proved, and thus is omitted.

Lemma 3.3. Suppose the failure rate function $r(t)$ is differentiable and strictly monotone on $\left(\tau_{1}, \tau_{2}\right)$. Let $\tau^{*} \in\left(\tau_{1}, \tau_{2}\right)$ be a critical point of $\mu(t)$, i.e., $\mu^{\prime}\left(\tau^{*}\right)=0$.

(i) If $r^{\prime}(t)>0, \forall t \in\left(\tau_{1}, \tau_{2}\right)$, then $\mu(t)$ has a bathtub shape in $\left(\tau_{1}, \tau_{2}\right)$ and achieves its minimum value on $\left[\tau_{1}, \tau_{2}\right]$ at $t=\tau^{*}$.

(ii) If $r^{\prime}(t)<0, \forall t \in\left(\tau_{1}, \tau_{2}\right)$, then $\mu(t)$ has an upside-down bathtub shape in $\left(\tau_{1}, \tau_{2}\right)$ and achieves its maximum value on $\left[\tau_{1}, \tau_{2}\right]$ at $t=\tau^{*}$.

In any case, $\mu(t)$ does not have any critical point in $\left(\tau_{1}, \tau_{2}\right)$ other than $\tau^{*}$.

Proof. We will first prove result (i). Result (ii) can be shown in a similar way. From equation (3.10), we see that $A^{\prime}(t)>0, \forall t \in\left(\tau_{1}, \tau_{2}\right)$. That is, $A(t)$ strictly increases in $t \in\left(\tau_{1}, \tau_{2}\right)$. Now, it is assumed that $\mu^{\prime}\left(\tau^{*}\right)=0$, so from (3.8), it follows that $A\left(\tau^{*}\right)=0$. Hence, $A(t)<0, \quad \forall \tau_{1}<t<\tau^{*}$, and $A(t)>0, \quad \forall \tau^{*}<t<\tau_{2}$. It further follows that $\mu^{\prime}(t)<0, \forall \tau_{1}<t<\tau^{*}$, and $\mu^{\prime}(t)>0, \forall \tau^{*}<t<\tau_{2}$, by (3.8). Therefore, $\mu(t)$ strictly decreases in $t \in\left(\tau_{1}, \tau^{*}\right]$, strictly increases in $t \in\left[\tau^{*}, \tau_{2}\right)$, has a bathtub shape on $\left(\tau_{1}, \tau_{2}\right)$, and achieves its minimum value on $\left[\tau_{1}, \tau_{2}\right]$ at $t=\tau^{*}$. 
Lemma 3.4. Suppose that in the interval $\left(\tau_{1}, \tau_{2}\right)$, a differentiable failure rate function $r(t)$ has a unique change point $\tau^{*} \in\left(\tau_{1}, \tau_{2}\right)$ and $\mu^{\prime}\left(\tau^{*}\right)=0$.

(i) If $r^{\prime}(t)<0$ on $\left(\tau_{1}, \tau^{*}\right)$ and $r^{\prime}(t)>0$ on $\left(\tau^{*}, \tau_{2}\right)$, then $\mu(t)$ strictly increases in $t \in\left(\tau_{1}, \tau_{2}\right)$

(ii) If $r^{\prime}(t)>0$ on $\left(\tau_{1}, \tau^{*}\right)$ and $r^{\prime}(t)<0$ on $\left(\tau^{*}, \tau_{2}\right)$, then $\mu(t)$ strictly decreases in $t \in\left(\tau_{1}, \tau_{2}\right)$.

Proof. Let us prove (i). The proof of (ii) is similar and will be omitted.

From (3.10), we see that $A^{\prime}(t)<0, \forall t \in\left(\tau_{1}, \tau^{*}\right)$. This implies that $A(t)$ strictly decreases in $t \in\left(\tau_{1}, \tau^{*}\right)$. Note that $A\left(\tau^{*}\right)=0$, since $\mu^{\prime}\left(\tau^{*}\right)=0$. Hence, $A(t)>$ $0, \forall t \in\left(\tau_{1}, \tau^{*}\right)$. This means that $\mu^{\prime}(t)>0, \forall t \in\left(\tau_{1}, \tau^{*}\right)$, or that $\mu(t)$ strictly increases in $t \in\left(\tau_{1}, \tau^{*}\right)$. On the other hand, $A^{\prime}(t)>0, \forall t \in\left(\tau^{*}, \tau_{2}\right)$, and so $A(t)$ strictly increases in $t \in\left(\tau^{*}, \tau_{2}\right)$. This, in turn, implies that $A(t)>0$, and so $\mu^{\prime}(t)>0, \quad \forall t \in\left(\tau^{*}, \tau_{2}\right)$. Consequently, $\mu(t)$ strictly increases in $t \in\left(\tau^{*}, \tau_{2}\right)$. Since $\mu(t)$ strictly increases both in $t \in\left(\tau_{1}, \tau^{*}\right)$ and in $t \in\left(\tau^{*}, \tau_{2}\right), \mu(t)$ strictly increases in $t \in\left(\tau_{1}, \tau_{2}\right)$

Theorem 3.5. Suppose the failure rate function $r(t)$ is differentiable and has rollercoaster shape with change points $\left\{t_{1}, \cdots, t_{k}\right\}$. Let $\mu(t)$ be the associated $M R L$ function. Then the following statements are true:

$1^{0} \mu(t)$ is strictly monotone on $\left[t_{k}, \infty\right)$ and $\mu(t)$ does not have $t_{k}$ as its change point. 
$2^{0} \mu(t)$ has at most one change point in each interval $\left(t_{j-1}, t_{j}\right), \quad 1 \leq j \leq k$, where $t_{0} \equiv 0$.

$3^{0}$ None of the $t_{j}, \quad 1 \leq j \leq k$, can be a change point of $\mu(t)$, even though it is a critical point of $\mu(t)$, i.e., it could hold that $\mu^{\prime}\left(t_{j}\right)=0$.

$4^{0} \mu(t)$ has at most $k$ change points and all of its change points must be in some of the open intervals $\left(t_{j-1}, t_{j}\right), \quad 1 \leq j \leq k$.

Proof. Result $1^{0}$ comes from Lemma 3.2. Applying Lemma 3.3, we obtain result $2^{0}$. To prove result $3^{0}$, we can assume $j \leq k-1$, since the case when $j=k$ is considered in $1^{0}$. Let $\tau_{1} \equiv t_{j-1}, \tau^{*}=t_{j}$, and $\tau_{2}=t_{j+1}$. Then, from Lemma 3.4 , we see that $t_{j}=\tau^{*}$ cannot be a change point of $\mu(t), \forall 1 \leq j \leq k-1$.

Finally, combining $1^{0}, 2^{0}$, and $3^{0}$, it follows that $4^{0}$ is also true.

Lemma 3.6. Assume that the failure rate function $r(t)$ is differentiable and strictly monotone on $\left(\tau_{1}, \tau_{2}\right)$.

(i) Suppose $\mu^{\prime}\left(\tau_{1}\right)=0$. If $r^{\prime}(t)>0, \forall t \in\left(\tau_{1}, \tau_{2}\right)$, then $\mu(t)$ strictly increases in $t \in\left[\tau_{1}, \tau_{2}\right] ;$ if $r^{\prime}(t)<0, \forall t \in\left(\tau_{1}, \tau_{2}\right)$, then $\mu(t)$ strictly decreases in $t \in\left[\tau_{1}, \tau_{2}\right]$.

(ii) Suppose $\mu^{\prime}\left(\tau_{2}\right)=0$. If $r^{\prime}(t)>0, \forall t \in\left(\tau_{1}, \tau_{2}\right)$, then $\mu(t)$ strictly decreases in $t \in\left[\tau_{1}, \tau_{2}\right] ;$ if $r^{\prime}(t)<0, \forall t \in\left(\tau_{1}, \tau_{2}\right)$, then $\mu(t)$ strictly increases in $t \in\left[\tau_{1}, \tau_{2}\right]$.

In particular, if $\mu^{\prime}\left(\tau_{1}\right)=0$, then $\mu^{\prime}\left(\tau_{2}\right) \neq 0$, and if $\mu^{\prime}\left(\tau_{2}\right)=0$, then $\mu^{\prime}\left(\tau_{1}\right) \neq 0$.

Proof. Let's first prove (i). Suppose $\mu^{\prime}\left(\tau_{1}\right)=0$ and $r^{\prime}(t)>0, \forall t \in\left(\tau_{1}, \tau_{2}\right)$. From equation (3.10) we see that $A^{\prime}(t)>0, \forall t \in\left(\tau_{1}, \tau_{2}\right)$. That is, $A(t)$ strictly increases in $t \in\left[\tau_{1}, \tau_{2}\right]$. It further implies that $A(t)>0, \forall t \in\left(\tau_{1}, \tau_{2}\right]$ since $A\left(\tau_{1}\right)=0$ by 
equation (3.8). Therefore, $\mu^{\prime}(t)>0, \forall t \in\left(\tau_{1}, \tau_{2}\right]$ from (3.8), which shows that $\mu(t)$ strictly increases in $t \in\left[\tau_{1}, \tau_{2}\right]$. The result when $r^{\prime}(t)<0, \quad \forall t \in\left(\tau_{1}, \tau_{2}\right)$, can be shown in a similar way.

Now, let's assume $\mu^{\prime}\left(\tau_{2}\right)=0$ and $r^{\prime}(t)>0, \quad \forall t \in\left(\tau_{1}, \tau_{2}\right)$. The assumption $\mu^{\prime}\left(\tau_{2}\right)=0$ yields $A\left(\tau_{2}\right)=0$ by equation(3.8). However, $r^{\prime}(t)>0, \forall t \in\left(\tau_{1}, \tau_{2}\right)$, implies $A^{\prime}(t)>$ $0, \forall t \in\left(\tau_{1}, \tau_{2}\right)$ from equation (3.10), i.e., $A(t)$ strictly increases in $t \in\left[\tau_{1}, \tau_{2}\right]$. This and the fact that $A\left(\tau_{2}\right)=0$ further show that $A(t)<0, \forall t \in\left[\tau_{1}, \tau_{2}\right)$. Therefore, $\mu^{\prime}(t)<0, \quad \forall t \in\left[\tau_{1}, \tau_{2}\right)$ by (3.8), i.e., $\mu(t)$ strictly decreases in $t \in\left[\tau_{1}, \tau_{2}\right]$. The case of $r^{\prime}(t)<0, \forall t \in\left(\tau_{1}, \tau_{2}\right)$, can be shown in the same way.

Remark 4. Let $r(t)$ be differentiable on $\left(\tau_{1}, \tau_{2}\right)$ and strictly monotone on $\left(\tau_{1}, \tau_{2}\right)$. Then $\mu(t)$ has at most one critical point on the closed interval $\left[\tau_{1}, \tau_{2}\right]$, by Lemmas 3.3 and 3.6 .

In Tang, Lu and Chew (1999), a sufficient condition is given for the MRL function to have a change point. The following result shows that this condition is also necessary.

Theorem 3.7. Suppose that $r(t)$ is a differentiable failure rate function and has a roller-coaster shape with change points $\left\{t_{1}, \cdots, t_{k}\right\}$. Let $\mu(t)$ be the associated $M R L$ function. The necessary and sufficient condition for $\mu(t)$ to have a change point in $\left(t_{j-1}, t_{j}\right), \quad 1 \leq j \leq k$, is

$$
\left[\mu\left(t_{j-1}\right) \cdot r\left(t_{j-1}\right)-1\right]\left[\mu\left(t_{j}\right) \cdot r\left(t_{j}\right)-1\right]<0 .
$$

Proof. We first show the sufficiency of condition (3.11). Note that

$$
\mu^{\prime}(t)=-1+r(t) \mu(t)
$$


Hence, condition (3.11) implies that $\mu^{\prime}\left(t_{j-1}\right) \mu^{\prime}\left(t_{j}\right)<0$. That is, $\mu^{\prime}\left(t_{j-1}\right)$ and $\mu^{\prime}\left(t_{j}\right)$ have different signs. Thus, there exist $t^{*} \in\left(t_{j-1}, t_{j}\right)$ such that $\mu^{\prime}\left(t^{*}\right)=0$. By Lemma $3.3, t^{*}$ must be a change point of $\mu(t)$. Also, from Lemma 3.3 , it follows that $t^{*}$ is the unique change point of $\mu(t)$ in $\left(t_{j-1}, t_{j}\right)$.

Now, we will prove the necessity of condition (3.11). If $\mu^{\prime}\left(t_{j-1}\right) \mu^{\prime}\left(t_{j}\right)=\left[\mu\left(t_{j-1}\right)\right.$. $\left.r\left(t_{j-1}\right)-1\right]\left[\mu\left(t_{j}\right) \cdot r\left(t_{j}\right)-1\right]=0$, whether $\mu^{\prime}\left(t_{j-1}\right)=0$ or $\mu^{\prime}\left(t_{j}\right)=0$, from Lemma 3.6 , we see that $\mu(t)$ is strictly monotone on $\left(t_{j-1}, t_{j}\right)$ and so cannot have any change points in $\left(t_{j-1}, t_{j}\right)$. If $\mu^{\prime}\left(t_{j-1}\right) \mu^{\prime}\left(t_{j}\right)>0$, then $\mu^{\prime}\left(t_{j-1}\right)$ and $\mu^{\prime}\left(t_{j}\right)$ have the same sign. For this interval $\left(t_{j-1}, t_{j}\right)$, let

$$
B \equiv\left\{t \in\left(t_{j-1}, t_{j}\right): \mu^{\prime}(t) \text { has the opposite sign of } \mu^{\prime}\left(t_{j-1}\right) \text { and } \mu^{\prime}\left(t_{j}\right)\right\} \text {. }
$$

We will show that $B=\varnothing$, i.e., $B$ is an empty set. Suppose the contrary is true. Then, if $B \neq \varnothing$ and $t^{*} \in B$, there exist $\tau^{*} \in\left(t_{j-1}, t^{*}\right)$ and $\tau^{* *} \in\left(t^{*}, t_{j}\right)$ such that $\mu^{\prime}\left(\tau^{*}\right)=\mu^{\prime}\left(\tau^{* *}\right)=0$. By Lemma 3.2 , both $\tau^{*}$ and $\tau^{* *}$ would be change points of $\mu(t)$ in $\left(t_{j-1}, t_{j}\right)$. But this contradicts result $2^{0}$ of Theorem 3.5. Hence, $B$ must be an empty set. Therefore, $\mu^{\prime}(t)$ keeps the same sign on $\left[t_{j-1}, t_{j}\right]$ and $\mu(t)$ cannot have any change point in $\left(t_{j-1}, t_{j}\right)$.

\subsection{Crossing of Failure Rate and MRL Functions}

Gupta and Gupta (2000) studied the crossing properties of two mean residual life functions in the case when the two associated failure rate functions cross at several points. It was shown there that the number of crossings of the mean residual life functions does not exceed the number of crossings of the failure rate functions. The 
location of these crossings was also examined in terms of the crossing points of the failure rate functions. However, there are some errors in their proof.

Let $F(t)$ and $G(t)$ be two lifetime distribution functions which have failure rate functions $\lambda(t)$ and $r(t)$, and mean residual life functions $\mu(t)$ and $\nu(t)$, respectively. To precisely develop our study, we first define the concept of a crossing point as follows.

Definition 2. Let $\varphi(t)$ and $\psi(t)$ be two functions. We say that $\varphi(t)$ and $\psi(t)$ have crossing points $\left\{-\infty<t_{1}<\cdots<t_{k}<\infty\right\}$ if $\triangle(t) \equiv \varphi(t)-\psi(t)$ has $t_{j}$ as the only zeros, does not change sign in each of the intervals $\left(t_{j-1}, t_{j}\right)$, and changes signs in any two adjacent intervals of the form $\left(t_{j-1}, t_{j}\right), \quad 1 \leq j \leq k+1$, where $t_{0} \equiv-\infty$ and $t_{k+1} \equiv \infty$.

Definition 3. Point $t^{*}$ is said to be a touching point of $\varphi(t)$ and $\psi(t)$ if $\triangle(t) \equiv$ $\varphi(t)-\psi(t)$ satisfies $\triangle\left(t^{*}\right)=0$.

Remark 5. According to the above definitions a crossing point must be a touching point, but the opposite is not always true.

With the above notation introduced, let us restate the result of Theorem 3.2 in Gupta and Gupta (2000) as follows : "Suppose $\lambda(t)$ and $r(t)$ have crossing points $0<t_{1}<\cdots<t_{k}<\infty$. Then $\mu(t)$ and $\nu(t)$ cross in at most $k$ points and at most one crossing point occurs in each of the intervals $\left(t_{j-1}, t_{j}\right), \quad 1 \leq j \leq k$, where $t_{0} \equiv 0$ ". This result is correct, but the proof provided there is incorrect. In case (iii) of the proof of their Theorem 3.2, they tried to show that $\mu\left(t_{j}\right) \neq \nu\left(t_{j}\right), \quad 1 \leq j \leq k$, by contradiction. Their proof goes as follows: 
"Suppose on the contrary $\mu\left(t_{j}\right)=\nu\left(t_{j}\right)$. Since $\lambda\left(t_{j}\right)=r\left(t_{j}\right)$, we have

$$
\frac{1+\mu^{\prime}\left(t_{j}\right)}{\mu\left(t_{j}\right)}=\frac{1+\nu^{\prime}\left(t_{j}\right)}{\nu\left(t_{j}\right)}
$$

The hypothesis implies that

$$
\mu^{\prime}\left(t_{j}\right)=\nu^{\prime}\left(t_{j}\right)
$$

or

$$
\lim _{h \rightarrow 0} \frac{\mu\left(t_{j}+h\right)-\nu\left(t_{j}+h\right)}{h}=0
$$

which is not possible because $\mu(t)<\nu(t)$ or $\mu(t)>\nu(t)$ on the right of $t_{j}$. Hence, $\mu\left(t_{j}\right) \neq \nu\left(t_{j}\right), \quad 1 \leq j \leq k$

The mistake is in assuming that if $\mu(t)<\nu(t)$ in a neighborhood to the right of $t_{j}$, this implies

$$
\frac{\mu\left(t_{j}+h\right)-\nu\left(t_{j}+h\right)}{h}<0
$$

if $h>0$ is sufficiently small, which further implies

$$
\lim _{h \rightarrow 0+} \frac{\mu\left(t_{j}+h\right)-\nu\left(t_{j}+h\right)}{h} \leq 0
$$

but this does not necessarily imply

$$
\lim _{h \rightarrow 0+} \frac{\mu\left(t_{j}+h\right)-\nu\left(t_{j}+h\right)}{h}<0
$$

However, they claimed

$$
\lim _{h \rightarrow 0+} \frac{\mu\left(t_{j}+h\right)-\nu\left(t_{j}+h\right)}{h}=0
$$

is impossible, which is wrong, and therefore, their proof is incorrect. Actually, not only this part of their proof is incorrect. The claim $\mu\left(t_{j}\right) \neq \nu\left(t_{j}\right)$, itself, made in 
their paper, is incorrect as well. Below we exhibit a counterexample in which two failure rate functions $r_{1}(t)$ and $r_{2}(t)$ have $t_{1}$ as their crossing point. In the meantime, their corresponding MRL functions $\mu_{1}(t)$ and $\mu_{2}(t)$ also have $t_{1}$ as a crossing point.

\section{Counterexample.}

Define $t_{1}=1$ and $t_{2}=2$, and let $0<h<\infty$ and $0<\varepsilon<1 / 2$ be two fixed numbers.

Let $0<c_{1}<\lambda<\infty$ and $0<d_{2}<d_{1}<\infty$.

On the interval $\left[t_{1}, \infty\right)=[1, \infty)$, we define the function $r_{1}(t)$ as follows: on the interval $[1+\varepsilon, 2-\varepsilon], r_{1}(t) \equiv c_{1}$; on the interval $[1,1+\varepsilon], r_{1}(t)$ is a linear function with $r_{1}(1)=h$ and $r_{1}(1+\varepsilon)=c_{1}$; on the interval $[2-\varepsilon, 2], r_{1}(t)$ is linear with $r_{1}(2-\varepsilon)=c_{1}$ and $r_{1}(2)=h$; on the interval $[2,2+\varepsilon], r_{1}(t)$ is again linear, $r_{1}(2)=h$, and $r_{1}(2+\varepsilon)=d_{1}$; and finally, $r_{1}(t)=d_{1}$ for all $t \geq 2+\varepsilon$. Let $r_{1}^{*}(t)>0$ be an arbitrary continuous function on $[0,1]$ satisfying $r_{1}^{*}(1)=h$. Then we can extend the domain of $r_{1}(t)$ to $[0, \infty)$ by defining $r_{1}(t)=r_{1}^{*}(t)$, for all $0 \leq t \leq 1$. Obviously, $r_{1}(t)>0$ is a continuous function on $[0, \infty)$. Summarizing the above, we have defined a continuous function $r_{1}(t)$ as 


$$
r_{1}(t)=\left\{\begin{array}{cc}
r_{1}^{*}(t), & \text { for } 0 \leq t \leq 1 ; \\
h, & \text { for } t=1 ; \\
h+\frac{c_{1}-h}{\varepsilon}(t-1), & \text { for } 1 \leq t \leq 1+\varepsilon ; \\
c_{1}, & \text { for } 1+\varepsilon \leq t \leq 2-\varepsilon ; \\
h+\frac{c_{1}-h}{\varepsilon}(2-t), & \text { for } 2-\varepsilon \leq t \leq 2 ; \\
h, & \text { for } t=2 ; \\
h+\frac{d_{1}-h}{\varepsilon}(t-2), & \text { for } 2 \leq t \leq 2+\varepsilon ; \\
d_{1}, & \text { for } t \geq 2+\varepsilon .
\end{array}\right.
$$

In a similar way, we can define a positive continuous function $r_{2}(t, \lambda)$ as

$$
r_{2}(t, \lambda)=\left\{\begin{array}{cc}
r_{2}^{*}(t), & \text { for } 0 \leq t \leq 1 ; \\
h, & \text { for } t=1 ; \\
h+\frac{\lambda-h}{\varepsilon}(t-1), & \text { for } 1 \leq t \leq 1+\varepsilon ; \\
\lambda, & \text { for } 1+\varepsilon \leq t \leq 2-\varepsilon ; \\
h+\frac{\lambda-h}{\varepsilon}(2-t), & \text { for } 2-\varepsilon \leq t \leq 2 ; \\
h, & \text { for } t=2 ; \\
h+\frac{d_{2}-h}{\varepsilon}(t-2), & \text { for } 2 \leq t \leq 2+\varepsilon ; \\
d_{2}, & \text { for } t \geq 2+\varepsilon ;
\end{array}\right.
$$

where $r_{2}^{*}(t)$ satisfies $0<r_{2}^{*}(t)<r_{1}^{*}(t)$ for all $0 \leq t<1$ and $r_{2}^{*}(1)=r_{1}^{*}(1)=h$. 
For any $\lambda>c_{1}>0$, we see the following relationships according to the above definitions:

$$
r_{1}(t) \quad\left\{\begin{array}{lc}
>r_{2}(t, \lambda), \quad \text { for } 0 \leq t<1 ; \\
=r_{2}(t, \lambda), \quad \text { for } t=1 ; \\
<r_{2}(t, \lambda), \quad \text { for } 1<t<2 ; \\
=r_{2}(t, \lambda), \quad \text { for } t=2 ; \\
>r_{2}(t, \lambda), \quad \text { for } t>2 .
\end{array}\right.
$$

Also, it is easy to see that $r_{1}(t)>0$ and $r_{2}(t, \lambda)>0$, for all $t \geq 0, \int_{0}^{\infty} r_{1}(t) d t=\infty$, and $\int_{0}^{\infty} r_{2}(t, \lambda) d t=\infty$. Therefore, both $r_{1}(t)$ and $r_{2}(t, \lambda)$ are failure rate functions. Further, they cross at exactly two points $t_{1}=1$ and $t_{2}=2$.

Denote the MRL function associated with the failure rate function $r_{2}(t, \lambda)$ by $\mu_{2}(t, \lambda)$. Then, by the definition of MRL, we have:

$$
\begin{aligned}
\mu_{2}\left(t_{1}, \lambda\right)=\mu_{2}(1, \lambda) & =\int_{0}^{\infty} e^{-\int_{1}^{1+x} r_{2}(u, \lambda) d u} d x \\
& =\int_{0}^{\varepsilon} e^{-\int_{1}^{1+x} r_{2}(u, \lambda) d u} d x+\int_{\varepsilon}^{\infty} e^{-\int_{1}^{1+x} r_{2}(u, \lambda) d u} d x
\end{aligned}
$$

Letting $\lambda \rightarrow \infty$ in the first integral, we have

$$
\begin{aligned}
\lim _{\lambda \rightarrow \infty} \int_{0}^{\varepsilon} e^{-\int_{1}^{1+x} r_{2}(u, \lambda) d u} d x & =\lim _{\lambda \rightarrow \infty} \int_{0}^{\varepsilon} e^{-\int_{1}^{1+x}\left[h+\frac{(\lambda-h)}{\varepsilon}(u-1)\right] d u} d x \\
& =\lim _{\lambda \rightarrow \infty} \int_{0}^{\varepsilon} e^{-\left[\left(\frac{\lambda-h}{2 \varepsilon}\right) x^{2}+h x\right]} d x=0
\end{aligned}
$$

As far as the second integral, similarly, we can obtain

$$
\lim _{\lambda \rightarrow \infty} \int_{\varepsilon}^{\infty} e^{-\int_{1}^{1+x} r_{2}(u, \lambda) d u} d x=\lim _{\lambda \rightarrow \infty} e^{-\int_{1}^{1+\varepsilon} r_{2}(u, \lambda) d u} \int_{\varepsilon}^{\infty} e^{-\int_{1+\varepsilon}^{1+x} r_{2}(u, \lambda) d u} d x=0
$$


Thus, it follows that $\lim _{\lambda \rightarrow \infty} \mu_{2}\left(t_{1}, \lambda\right)=0$.

On the other hand, denote $\lim _{\lambda \rightarrow c_{1}} r_{2}(t, \lambda)$ as $r_{2}\left(t, c_{1}\right)$. Then

$$
r_{2}\left(t, c_{1}\right) \quad\left\{\begin{array}{lc}
=r_{1}(t) & \text { for } t_{1} \leq t \leq t_{2} \\
<r_{1}(t) & \text { for } t>t_{2}
\end{array}\right.
$$

Hence, $\mu_{2}\left(t_{1}, c_{1}\right)=\lim _{\lambda \rightarrow c_{1}} \mu_{2}\left(t_{1}, \lambda\right)$ and $\mu_{2}\left(t_{1}, c_{1}\right)>\mu_{1}\left(t_{1}\right)$.

Summarizing the above, we have shown that $\mu_{2}\left(t_{1}, \infty\right)=0<\mu_{1}\left(t_{1}\right)<\mu_{2}\left(t_{1}, c_{1}\right)$.

Note that $\mu_{2}(t, \lambda)$ is a continuous function of $\lambda>0$. Therefore, there exists $c_{1}<\lambda^{*}<$ $\infty$ such that $\mu_{2}\left(t_{1}, \lambda^{*}\right)=\mu_{1}\left(t_{1}\right)$.

Now, we define

$$
r_{2}(t)=\left\{\begin{array}{lc}
r_{2}{ }^{*}(t), & \text { if } \quad 0 \leq t \leq 1 \\
r_{2}\left(t, \lambda^{*}\right), & \text { if } t>1
\end{array}\right.
$$

Then $r_{1}(t)$ and $r_{2}(t)$ have exactly two crossing points, $t_{1}=1$ and $t_{2}=2$, and it holds that $\mu_{2}\left(t_{1}\right)=\mu_{1}\left(t_{1}\right)$. This example shows that the claim made in (iii) of the proof of Theorem 3.2 in Gupta and Gupta (2000) is incorrect.

In the rest of this section, we will revisit Theorem 3.2 in Gupta and Gupta (2000) and give a new proof.

Lemma 3.8. Suppose that on the interval $\left(\tau_{1}, \tau_{2}\right)$, the failure rate functions $\lambda(t)$ and $r(t)$ satisfy $\lambda(t)<r(t), \quad \forall t \in\left(\tau_{1}, \tau_{2}\right)$. Then

(i) If $\mu\left(\tau_{2}\right)=\nu\left(\tau_{2}\right)$, then $\mu(t) \neq \nu(t), \forall t \in\left[\tau_{1}, \tau_{2}\right)$;

(ii) If $\mu\left(\tau_{1}\right)=\nu\left(\tau_{1}\right)$, then $\mu(t) \neq \nu(t), \forall t \in\left(\tau_{1}, \tau_{2}\right]$. 
Proof. For any given $0 \leq x_{1}<x_{2}$, we have

$$
\begin{aligned}
\mu\left(x_{1}\right) & =\frac{\int_{x_{1}}^{\infty} \bar{F}(t) d t}{\bar{F}\left(x_{1}\right)}=\int_{0}^{\infty} \frac{\bar{F}\left(x_{1}+x\right)}{\bar{F}\left(x_{1}\right)} d x=\int_{0}^{\infty} e^{-\int_{x_{1}}^{x_{1}+x} \lambda(u) d u} d x \\
& =\int_{0}^{x_{2}-x_{1}} e^{-\int_{x_{1}}^{x_{1}+x} \lambda(u) d u} d x+\int_{x_{2}-x_{1}}^{\infty} e^{-\int_{x_{1}}^{x_{2}} \lambda(u) d u-\int_{x_{2}}^{x_{1}+x} \lambda(u) d u} d x \\
& =\int_{0}^{x_{2}-x_{1}} e^{-\int_{x_{1}}^{x_{1}+x} \lambda(u) d u} d x+e^{-\int_{x_{1}}^{x_{2}} \lambda(u) d u} \int_{x_{2}-x_{1}}^{\infty} e^{-\int_{x_{2}}^{x_{1}+x} \lambda(u) d u} d x \\
& =\int_{0}^{x_{2}-x_{1}} e^{-\int_{x_{1}}^{x_{1}+x} \lambda(u) d u} d x+e^{-\int_{x_{1}}^{x_{2}} \lambda(u) d u} \int_{0}^{\infty} e^{-\int_{x_{2}}^{x_{2}+y} \lambda(u) d u} d y \\
& =\int_{0}^{x_{2}-x_{1}} e^{-\int_{x_{1}}^{x_{1}+x} \lambda(u) d u} d x+\mu\left(x_{2}\right) e^{-\int_{x_{1}}^{x_{2}} \lambda(u) d u}
\end{aligned}
$$

Similarly, we have

$$
\nu\left(x_{1}\right)=\int_{0}^{x_{2}-x_{1}} e^{-\int_{x_{1}}^{x_{1}+x} r(u) d u} d x+\nu\left(x_{2}\right) e^{-\int_{x_{1}}^{x_{2}} r(u) d u} .
$$

Now, if $\mu\left(\tau_{2}\right)=\nu\left(\tau_{2}\right)$, then for any $t \in\left[\tau_{1}, \tau_{2}\right)$ from (3.15) and (3.16) we see that

$$
\begin{aligned}
\mu(t) & =\int_{0}^{\tau_{2}-t} e^{-\int_{t}^{t+x} \lambda(u) d u} d x+\mu\left(\tau_{2}\right) e^{-\int_{t}^{\tau_{2}} \lambda(u) d u} \\
& >\int_{0}^{\tau_{2}-t} e^{-\int_{t}^{t+x} \lambda(u) d u} d x+\nu\left(\tau_{2}\right) e^{-\int_{t}^{\tau_{2}} r(u) d u} \\
& >\int_{0}^{\tau_{2}-t} e^{-\int_{t}^{t+x} r(u) d u} d x+\nu\left(\tau_{2}\right) e^{-\int_{t}^{\tau_{2}} r(u) d u}=\nu(t) .
\end{aligned}
$$

Therefore, (i) holds.

Result (ii) can be shown in the same way and is thus omitted.

Theorem 3.9. Let $F(t)$ and $G(t)$ be two lifetime distributions. Suppose that $F(t)$ and $G(t)$ have failure rate functions $\lambda(t)$ and $r(t)$, and mean residual life functions $\mu(t)$ 
and $\nu(t)$, respectively. If $\lambda(t)$ and $r(t)$ have crossing points $\left\{0<t_{1}<\cdots<t_{k}<\infty\right\}$, then

(i) $\mu(t)$ and $\nu(t)$ do not have a touching point on $\left[t_{k}, \infty\right)$;

(ii) $\mu(t)$ and $\nu(t)$ have at most one touching point on each $\left[t_{j-1}, t_{j}\right], \quad 1 \leq j \leq k$;

(iii) $\mu(t)$ and $\nu(t)$ have at most $k$ touching points, and so they have at most $k$ crossing points. Also, these crossing points must be in open intervals $\left(t_{j-1}, t_{j}\right)$, where $1 \leq j \leq k$.

Proof. Result (i) was proved in Theorem 3.2 of Gupta and Gupta (2000).

To show (ii), let $t^{*} \in\left[t_{j-1}, t_{j}\right]$ and $\mu\left(t^{*}\right)=\nu\left(t^{*}\right)$. Applying Lemma 3.8 to the interval $\left[t_{j-1}, t^{*}\right]$, we see that $\mu(t) \neq \nu(t), \quad \forall t \in\left[t_{j-1}, t^{*}\right)$. Similarly, applying Lemma 3.8 to $\left[t^{*}, t_{j}\right]$, we see that $\mu(t) \neq \nu(t), \quad \forall t \in\left(t^{*}, t_{j}\right]$. Thus, $\mu(t) \neq \nu(t), \quad \forall \quad t \in$ $\left[t_{j-1}, t_{j}\right] \backslash\left\{t^{*}\right\}$. This shows that $\mu(t)$ and $\nu(t)$ have at most one touching point on $\left[t_{j-1}, t_{j}\right]$

Result (iii) follows from (i) and (ii).

\section{Concluding Remarks}

In this study, we discussed the behaviors of the MRL function for both continuous and discrete lifetime distributions with respect to their failure rate functions and vise versa, and established a new result for the relationship between discrete bathtubshaped MRL and upside-down bathtub-shaped failure rate functions. The study showed that if a discrete MRL has a bathtub shape, then under some conditions the corresponding failure rate function has an upside-down bathtub shape. Some mistakes in the proofs of Tang, Lu and Chew (1999) were corrected, and a new 
necessary and sufficient condition for the MRL to follow the roller-coaster behavior of the corresponding failure rate function was also derived. This study also corrected some mistakes in the proofs in Gupta and Gupta (2000), with the ensuing results being expanded and proved thoroughly to establish the relationship between the crossing points of the failure rate and associated MRL functions.

The new results derived in this study will be useful to model various lifetime data that occur in environmental studies, medical research, electronics engineering, and in many other areas of science and technology. 


\section{List of References}

1. Ghai, G.L. and Mi, J. (1999). "Mean Residual Life and Its Association With Failure Rate", IEEE Trans. Reliability, vol. 48, 262-266.

2. Gupta, R.C. (1981). "On the mean residual life function in survival studies", Statistical Distributions in Scientific Work, vol 5, 327-334.

3. Gupta, R.C. and Gupta, P.L. (2000). "On the crossing of reliability measures", Statistics and Probability Letters, 46, 301-305.

4. Mi, J. (1993). "Discrete bathtub failure rate and upside-down bathtub mean residual life", Naval Research Logistics, vol 40, 361-371.

5. Mi, J. (1995). "Bathtub failure rate and upside-down bathtub mean residual life", IEEE Trans. Reliability, vol. 44, 388-391.

6. Mi, J. (1996). "Minimizing Some Cost Functions Related to Both Burn-In and Field Use", Operations Research, vol. 49, 497-500.

7. Peck, D.S. and Zierdt, C.H. (1974), "The Reliability of Semiconductor Devices in the Bell System", Proceeding of the IEEE, vol. 62, 185-211.

8. Tang, L.C., Lu, Y. and Chew, E.P. (1999). "Mean Residual Life of Lifetime Distributions", IEEE Trans. Reliability, vol. 48, 73-78.

9. Wong, L.K. (1988). "The bathtub does not hold water any more", Quality and Reliability Engineering Int'l, vol.4, 279-286.

10. Wong, L.K. (1989). "The roller-coaster curve is in", Quality and Reliability Engineering Int'l, vol.5, 29-36.

11. Wong, L.K. (1991). "The Physical basis for the roller-coaster hazard rate curve for electronics", Quality and Reliability Engineering Int'l, vol.7, 489-495. 